\title{
EFECTOS DE LA PERTURBACIÓN ANTRÓPICA EN PETENES DE SELVA EN CAMPECHE, MÉXICO
}

\author{
Luz Gabriela Koyoc-Ramírez ${ }^{1}$, Jorge Mendoza-VegaA ${ }^{1,3}$, Juan Carlos Pérez \\ JiMÉNEZ ${ }^{1}$ Y NURIA TORRESCANO VALLE ${ }^{2}$
}

\begin{abstract}
${ }^{1}$ El Colegio de la Frontera Sur (ECOSUR), Unidad Campeche, Avenida Rancho, Polígono 2-A, Parque Industrial Lerma, 24500 Campeche, Campeche, México. ${ }^{2}$ El Colegio de la Frontera Sur (ECOSUR), Unidad Chetumal, Avenida Centenario km 5.5, 77014 Chetumal, Quintana Roo, México.

${ }^{3}$ Autor para la correspondencia: jmendoza@ecosur.mx
\end{abstract}

\section{RESUMEN}

Los petenes son islas naturales de vegetación, únicos en su tipo a nivel mundial, restringidos a la Península de Yucatán, México, los Everglades en Florida, Estados Unidos de América, y la Ciénaga de Zapata, Cuba. En México, este ecosistema ha estado sujeto a perturbación antrópica, principalmente debido a la extracción de madera, la fragmentación, incendios, extracción de flora y fauna, y la expansión de asentamientos humanos. No obstante su importancia ecológica, existen pocos estudios acerca de su estado actual. En el presente trabajo se analizó el efecto de la intervención humana sobre la vegetación y las propiedades del suelo en petenes de la Reserva de la Biosfera Los Petenes, Campeche, comparando tres petenes perturbados y tres conservados. Los conservados tuvieron menor densidad de plantas (ind/ha) en todos los estratos, menor cobertura vegetal (en los estratos en que se midió; medio e inferior) $\left(\mathrm{m}^{2} / \mathrm{ha}\right)$, y mayor área basal (medida sólo en el estrato superior) ( $\mathrm{m}^{2} / \mathrm{ha}$ ). Los suelos no difirieron entre las dos clases de petenes, lo que indica que las actividades antrópicas no han causado un impacto negativo en ellos.

Palabras clave: características del suelo, estratos de vegetación, islas de vegetación, petenes conservados, petenes perturbados.

\section{ABSTRACT}

Petenes (hammock ecosystems) are island-like natural spots of vegetation, unique worldwide, restricted to the Peninsula of Yucatan, Mexico, the Everglades in Florida, United 
States of America, and the Ciénaga de Zapata, Cuba. In Mexico, this ecosystem has been subject to anthropogenic disturbance, mainly due to wood extraction, land fragmentation, fires, flora and fauna extraction, and the expansion of human settlements. Notwithstanding its ecological importance, there are few studies concerning its current status. In the present work the effect of human intervention upon the vegetation and soil properties in petenes of the Petenes Biosphere Reserve, Campeche was analysed, comparing three disturbed and three conserved petenes. The conserved petenes had lower plant density (ind/ha) in all vegetation layers, lower plant cover (in the measured layers: medium and lower) $\left(\mathrm{m}^{2} / \mathrm{ha}\right)$, and higher basal area (measured only in the superior layer) $\left(\mathrm{m}^{2} / \mathrm{ha}\right)$. Soils did not differ between the two classes of petenes, which suggests that the antropic activities have not caused a negative impact on them.

Key words: conserved petenes, disturbed petenes, soil characteristics, vegetation islands, vegetation layers.

\section{INTRODUCCIÓN}

México se ubica entre los países más diversos a nivel mundial. En contraste, las actividades antrópicas están provocando la degradación de los bosques tropicales del país, lo cual induce a la pérdida de la diversidad biológica (Chapin et al., 2000). Entre 1993 y 2002 se registró en el país una tasa de deforestación de $0.25 \%$ por año; la región sureste alcanzó los valores más altos con $0.76 \%$ (Bray et al., 2004). La Península de Yucatán, provincia florística tropical con asociaciones vegetales restringidas a condiciones ecológicas particulares, ha sufrido altas tasas de deforestación, lo que significó una reducción de más de un millón de hectáreas de sus selvas de 1985 a 1994 (Turner et al., 2001). Solo en el estado de Campeche, de 2003 a 2009, se deforestaron 38,784 hectáreas de selvas por año.

La pérdida de diversidad biológica y servicios ambientales en la Península, se asocia principalmente a fenómenos naturales como los huracanes y a actividades humanas como el cambio de uso de suelo (Turner et al., 2001). Los petenes son islas naturales de vegetación arbórea (manglar y selva) inmersas en una matriz de vegetación inundable de ciénagas someras y pantanosas (Durán, 1987). Estos ecosistemas han sido encontrados exclusivamente en la Península de Yucatán, México, los Everglades de Florida, Estados Unidos de América y la Ciénaga de Zapata, Cuba (Olmsted et al., 1980). Debido a su importancia ecológica y social, la región donde se ubican los petenes en el estado de Campeche fue decretada Reserva de la Biósfera Los Petenes (RBLP) e incluida en 2004 en la lista de humedales RAMSAR. 
Los estudios realizados en la región se han enfocado principalmente en el análisis de la estructura y riqueza de especies vegetales (Durán, 1987). Rico-Gray (1982) reveló además aspectos hidrológicos (salinidad e inundación) así como rasgos de la complejidad en la composición de especies.

La mayor perturbación antrópica en los petenes de la Península de Yucatán se remonta a la época colonial con la extracción de maderas preciosas: caoba (Swietenia macrophyla) y cedro (Cedrela odorata) y de hojas de palma de huano (Sabal japa). A partir de los años 80 del siglo pasado se intensificó la extracción selectiva de especies maderables, provocando la disminución del número de especies y promoviendo el desarrollo de numerosas áreas de vegetación secundaria (Rico-Gray, 1982). En la actualidad, la agricultura, ganadería y el crecimiento urbano en la periferia de la RBLP constituyen una fuerte presión al área natural protegida. Los pobladores realizan cacería furtiva y la extracción ilegal de diversas especies vegetales como el huano (Sabal mexicana) y el zapote (Manilkara zapota). Por otro lado, 16\% de la superficie de la RBLP ha sufrido incendios inducidos (Mas y Correa Sandoval, 1999).

Por lo anterior, el disturbio causado por el hombre en los petenes de selva se considera como una gran amenaza a la diversidad, integridad y funcionalidad de asociaciones vegetales de alta importancia ecológica y su dinámica espacio-temporal (Chapin et al., 2000). El objetivo del presente estudio fue identificar los efectos de la perturbación antrópica por medio de la comparación de la estructura, composición, riqueza y diversidad vegetal y las características del suelo entre petenes de selva perturbados y conservados en la RBLP.

\section{MATERIALES Y MÉTODOS}

La RBLP es una franja costera ubicada a $20^{\circ} 51^{\prime} 30^{\prime \prime}$ y $19^{\circ} 49^{\prime} 00^{\prime \prime}$ de latitud norte y $90^{\circ} 45^{\prime} 15^{\prime \prime}$ y $90^{\circ} 20^{\prime} 00^{\prime \prime}$ de longitud oeste, cuya extensión es de 282857 hectáreas. Limita al norte con la Reserva de la Biosfera Ría Celestún y Golfo de México, al oeste con el Golfo de México, al este con los municipios de Calkini, Hecelchacán, Tenabo y Campeche, y al sur con el municipio de Campeche. Forma parte de la ecoregión Los Petenes - Celestún - El Palmar, que en conjunto abarcan 418888 hectáreas. El clima en la zona de estudio es cálido subhúmedo con lluvias en verano (Aw). La temperatura y precipitación media anual, de acuerdo con la Comisión Nacional de Áreas Naturales Protegidas (CONANP), es de $26.4{ }^{\circ} \mathrm{C}$ y $1049.7 \mathrm{~mm}$ respectivamente. Se identifican dos épocas climáticas para la región: la temporada de secas que abarca de noviembre a abril y la de lluvias, de mayo a octubre. Existe 
además un período de sequía intraestival (canícula) durante lo más acentuado de las lluvias (Rico-Gray, 1982).

En la Reserva, el agua subterránea que corre hacia el Golfo de México aflora a través de ojos de agua y canales naturales que interconectan a los petenes; el flujo proviene de la planicie interior donde las inclinaciones del terreno son menores de $0.5 \%$ y las rocas son de origen calizo sedimentario. Entre los suelos destacan los regosoles, histosoles, rendzinas y gleysoles.

La vegetación incluye manglares, selva mediana y baja subcaducifolia (Mas y Correa Sandoval, 1999). Los petenes estudiados conforman círculos concéntricos que al exterior presentan especies de mangle (Flores y Espejel, 1994) y al interior elementos arbóreos dispersos de Manilkara zapota, Sabal japa, Metopium brownei, Gymnanthes lucida, entre otros (Flores y Espejel, 1994; Zamora, 2003). En el estrato medio e inferior destacan Bravaisia berlandieriana, Acrostichum aureum y Sabal japa (Rico-Gray, 1982; Flores y Espejel, 1994; Zamora, 2003).

Por medio de la interpretación de ortofotos y posteriormente recorridos de campo se seleccionaron los sitios de muestreo: petenes de selva $(\geq 500 \mathrm{~m}$ de diámetro) conservados y perturbados, los cuales se ubicaron hacia la parte central, en relación norte-sur del área núcleo de la RBLP (Fig. 1). La franja de petenes de selva se encuentra más cerca del límite de la reserva hacia tierra adentro que de la costa.

Los petenes fueron diferenciados con base en el grado de perturbación a la que han estado sujetos:

Los conservados ( $\mathrm{C} 1, \mathrm{C} 2$ y $\mathrm{C} 3)$ fueron elegidos de acuerdo con los siguientes criterios: 1) tener una vegetación de selva mediana subperennifolia bien conservada, 2) estar ubicados lejos de caminos y poblaciones humanas y por lo tanto de difícil acceso en cualquier época del año y 3) la perturbación, en caso de existir, que fuera de carácter natural (árboles caídos y muertos en pie).

Los perturbados (P1, P2 y P3) fueron escogidos de la siguiente manera: 1) tener una vegetación de selva mediana subperennifolia con evidencias de afectación humana, tales como aprovechamiento de especies (flora y fauna), incendios, senderos, 2) cercanos a poblaciones humanas y de fácil acceso, y 3) ser atravesados por un camino de terraplén.

Para el muestreo de vegetación se delimitó un transecto en banda, de norte a sur, siguiendo el método de Gentry (1988), pero usando longitudes variables a partir del tamaño de cada peten. El ancho del transecto fue de $2 \mathrm{~m}$, el área mínima de muestreo fue de $1000 \mathrm{~m}^{2}$ (C2) y la máxima de $2800 \mathrm{~m}^{2}$ (C3). La longitud del transecto se determinó por el cambio fisonómico de vegetación: de árboles de selva a árboles de manglar. Para cubrir los distintos estratos y formas de vida, los levantamien- 


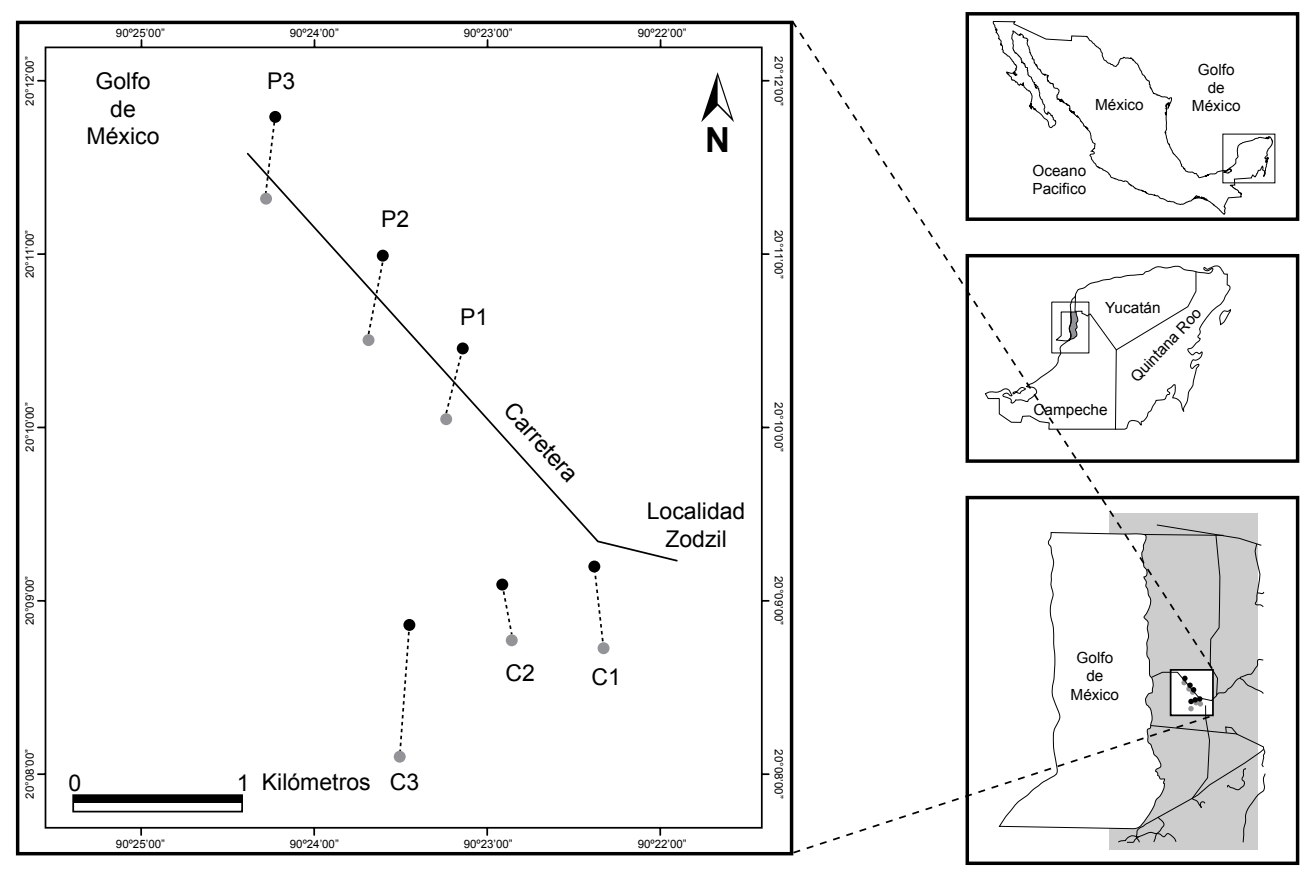

Fig. 1. Ubicación de los petenes seleccionados: conservados (C) y perturbados (P) en la Reserva de la Biósfera Los Petenes, Campeche, México. Los puntos delimitan la longitud del transecto de muestreo.

tos de vegetación se hicieron en subparcelas de $10 \times 2 \mathrm{~m}$, en las cuales se realizó una caracterización general de cada sitio. Además se registraron todos los individuos en las distintas formas de vida y se determinó su pertenencia a tres estratos: superior ( $\geq 3 \mathrm{~m}$ altura), medio $(<3 \mathrm{~m}$ pero $\geq 1 \mathrm{~m}$ ) e inferior $(<1 \mathrm{~m})$. Para cada individuo se anotó a) nombre común; b) diámetro a la altura del pecho (DAP); c) altura total; d) cobertura, a partir de dos medidas de la copa (longitudinal y transversal); y e) se colectó material vegetal para la determinación de la identidad botánica.

Con los datos registrados se calcularon la densidad (número de individuos por hectárea), el área basal $(\mathrm{AB})$ del estrato superior mediante la ecuación $\mathrm{AB}=$ $\left(\mathrm{d}^{2} / 4\right) \mathrm{p}$, donde $\mathrm{d}=$ diámetro, y la cobertura de los estratos medio e inferior como la proyección horizontal de la copa utilizando el diámetro promedio (DPC) mediante la ecuación: Cobertura $=\mathrm{p} / 4(\mathrm{DPC})^{2}$. También se estimó la diversidad de especies con el índice de Shannon-Wiener: $H^{\prime}=-\sum$ pi ln pi, donde: $p i$ es la abundancia proporcional de las especies $=(\mathrm{ni} / \mathrm{N}), i=1 \ldots n, n$ es el número de especies, $\mathrm{N}$ es el número de 
registros. Para comparar entre los tipos de petenes se usó la prueba $t$ de Hutcheson (Magurran, 2006).

Las diferencias entre petenes perturbados y conservados en densidad, área basal y cobertura se estudiaron mediante una prueba $t$ para dos muestras. Cuando no se cumplieron los supuestos de normalidad y homogeneidad de varianzas, se realizó la prueba no paramétrica Mann-Whitney.

En el transecto definido para muestreo de vegetación se abrieron pozos pedológicos (1.5 x $2 \mathrm{~m}$ y $1 \mathrm{~m}$ de profundidad o hasta encontrar roca), utilizando la metodología de la FAO (Anónimo, 2009), uno en el borde sur (transición selva-manglar) y uno más al centro de cada transecto (selva).

La descripción de los suelos se hizo de acuerdo con la norma de la International Union of Soil Sciences (Anónimo, 2007) y se tomaron muestras (en total 58) de cada uno de los horizontes identificados para determinar color (Tabla Munsell), densidad aparente (cilindros de $5 \mathrm{~cm}$ de diámetro X $5 \mathrm{~cm}$ de largo), y de acuerdo con el manual de procedimientos para análisis de suelos de la International Soil Reference and Information Centre (Anónimo, 2002), se determinó (pH (relación 1:2 en agua), porcentaje de carbonatos (neutralización ácida), carbono orgánico (Walkley y Black), nitrógeno total (semi-micro kjeldhal), fósforo (Olsen para suelos neutros y alcalinos) y capacidad de intercambio catiónico (acetato de amonio a $1 \mathrm{~N} \mathrm{y} \mathrm{pH} \mathrm{7).} \mathrm{Los}$ resultados de los perfiles de suelo, para cada una de las características estudiadas y horizonte se compararon por medio de un análisis de varianza y la prueba KruskalWallis cuando los datos no cumplieron los supuestos de normalidad.

\section{RESULTADOS}

\section{Vegetación}

Se registró un total de 30 especies, distribuidas en 23 familias (Cuadro 1). Los petenes perturbados tuvieron una densidad promedio de $5422 \mathrm{ind} / \mathrm{ha}$, mientras que los conservados de $2455.2 \mathrm{ind} / \mathrm{ha}$ para los tres estratos considerados. De acuerdo con el índice de Shannon-Wiener y la comparación por la prueba $t$ de Hucheson, los valores de diversidad para ambos tipos de peten son muy similares. Solo se encontraron diferencias significativas $(\mathrm{p}<0.01)$ entre los valores del estrato inferior: 1.024 y de 1.507 para los petenes perturbados y conservados respectivamente. En los conservados dominaron en el estrato superior las familias Anacardiaceae, Sapotaceae y Euphorbiaceae, mientras que en los perturbados lo hicieron Fabaceae, Mimosaceae, Anacardiaceae y Acanthaceae. 
Cuadro 1. Presencia de las especies encontradas en los diferentes estratos de petenes con distinto grado de disturbio. Una $\mathrm{X}$ indica su presencia.

\begin{tabular}{|c|c|c|c|c|c|c|c|}
\hline \multirow[t]{2}{*}{ Familia } & \multirow[t]{2}{*}{ Especie } & \multicolumn{2}{|c|}{ Superior } & \multicolumn{2}{|c|}{ Medio } & \multicolumn{2}{|c|}{ Inferior } \\
\hline & & $\mathrm{C}$ & $\mathrm{P}$ & $\mathrm{C}$ & $P$ & $\mathrm{C}$ & $\mathrm{P}$ \\
\hline Acanthaceae & $\begin{array}{l}\text { Bravaisia berlandieriana (Nees) T.F. } \\
\text { Daniel }\end{array}$ & $\mathrm{X}$ & $\mathrm{X}$ & $\mathrm{X}$ & $\mathrm{X}$ & $\mathrm{X}$ & $\mathrm{X}$ \\
\hline Anacardiaceae & Metopium brownei Urb. & $\mathrm{X}$ & $\mathrm{X}$ & $\mathrm{X}$ & $\mathrm{X}$ & $\mathrm{X}$ & $\mathrm{X}$ \\
\hline Arecaceae & Sabal japa C. Wright ex Bartlett & $\mathrm{X}$ & $\mathrm{X}$ & $\mathrm{X}$ & $\mathrm{X}$ & $\mathrm{X}$ & $\mathrm{X}$ \\
\hline Arecaceae & Sabal mexicana Mart. & $\mathrm{X}$ & $\mathrm{X}$ & - & - & $\mathrm{X}$ & - \\
\hline Burseraceae & Bursera simaruba (L.) Sarg. & $\mathrm{X}$ & $\mathrm{X}$ & $\mathrm{X}$ & $\mathrm{X}$ & $\mathrm{X}$ & $\mathrm{X}$ \\
\hline Combretaceae & Conocarpus erectus $\mathrm{L}$. & $\mathrm{X}$ & $\mathrm{X}$ & - & - & - & - \\
\hline Cyperaceae & Cladium jamaicense Crantz & - & - & $\mathrm{X}$ & $\mathrm{X}$ & $\mathrm{X}$ & $\mathrm{X}$ \\
\hline Ebenaceae & Diospyros verae-crucis (Standl.) Standl & $\mathrm{X}$ & $\mathrm{X}$ & $\mathrm{X}$ & $\mathrm{X}$ & $\mathrm{X}$ & $\mathrm{X}$ \\
\hline Ebenaceae & Diospyros yatesiana Standl. & $\mathrm{X}$ & $\mathrm{X}$ & $\mathrm{X}$ & $\mathrm{X}$ & $\mathrm{X}$ & - \\
\hline Erythroxylaceae & Erythroxylum areolatum $\mathrm{L}$. & $\mathrm{X}$ & $\mathrm{X}$ & $\mathrm{X}$ & $\mathrm{X}$ & $\mathrm{X}$ & $\mathrm{X}$ \\
\hline Euphorbiaceae & Gymnanthes lucida Sw. & $\mathrm{X}$ & $\mathrm{X}$ & $\mathrm{X}$ & $\mathrm{X}$ & $\mathrm{X}$ & $\mathrm{X}$ \\
\hline Fabaceae & Erythrina standleyana Krukoff & $\mathrm{X}$ & - & - & - & $\mathrm{X}$ & - \\
\hline Fabaceae & Piscidia piscipula (L.) Sarg. & $\mathrm{X}$ & $\mathrm{X}$ & $\mathrm{X}$ & $\mathrm{X}$ & $\mathrm{X}$ & $\mathrm{X}$ \\
\hline Leguminosae & Lonchocarpus rugosus Benth. & - & - & $\mathrm{X}$ & - & $\mathrm{X}$ & - \\
\hline Malvaceae & Hampea trilobata Standl. & $\mathrm{X}$ & $\mathrm{X}$ & $\mathrm{X}$ & $\mathrm{X}$ & $\mathrm{X}$ & $\mathrm{X}$ \\
\hline Malvaceae & Malvaviscus arboreus Cav. & - & - & $\mathrm{X}$ & $\mathrm{X}$ & $X$ & $\mathrm{X}$ \\
\hline Meliaceae & Swietenia macrophylla King in Hook. & $\mathrm{X}$ & - & $\mathrm{X}$ & $\mathrm{X}$ & $\mathrm{X}$ & $\mathrm{X}$ \\
\hline Mimosaceae & Lysiloma bahamensis Benth. & $\mathrm{X}$ & $\mathrm{X}$ & - & - & - & $\mathrm{X}$ \\
\hline Mimosaceae & Lysiloma latisiliquum (L.) Benth. & $\mathrm{X}$ & $\mathrm{X}$ & - & - & - & $\mathrm{X}$ \\
\hline Moraceae & Ficus cotinifolia Kunth & $\mathrm{X}$ & $\mathrm{X}$ & - & - & - & - \\
\hline Moraceae & Ficus obtusifolia Kunth & $\mathrm{X}$ & $\mathrm{X}$ & - & - & $\mathrm{X}$ & $\mathrm{X}$ \\
\hline Polygonaceae & Coccoloba cozumelensis Hemsl. & - & $\mathrm{X}$ & - & - & $\mathrm{X}$ & $\mathrm{X}$ \\
\hline Polypodiaceae & $\begin{array}{l}\text { Acrostichum daneaefolium Langsd. \& } \\
\text { Fisch. }\end{array}$ & - & - & - & $\mathrm{X}$ & - & $\mathrm{X}$ \\
\hline Rhamnaceae & Krugiodendron ferreum Urb. & $\mathrm{X}$ & $\mathrm{X}$ & - & - & $\mathrm{X}$ & $\mathrm{X}$ \\
\hline Rhizophoraceae & Rhizophora mangle $\mathrm{L}$. & $\mathrm{X}$ & $\mathrm{X}$ & - & - & - & - \\
\hline Salicaceae & Zuelania guidonia (Sw.) Britton \& Millsp. & - & $\mathrm{X}$ & - & - & $\mathrm{X}$ & - \\
\hline Sapindaceae & Talisia olivaeformis (Kunth) Radlk. & $\mathrm{X}$ & $\mathrm{X}$ & $\mathrm{X}$ & - & $\mathrm{X}$ & $\mathrm{X}$ \\
\hline Sapotaceae & Manilkara zapota (L.) P. Royen & $\mathrm{X}$ & $\mathrm{X}$ & $\mathrm{X}$ & $\mathrm{X}$ & $\mathrm{X}$ & $\mathrm{X}$ \\
\hline Theophrastaceae & Jacquinia aurantiaca Ait. & - & - & $\mathrm{X}$ & $\mathrm{X}$ & $\mathrm{X}$ & - \\
\hline Verbenaceae & Avicennia germinans $(\mathrm{L}.) \mathrm{L}$. & $\mathrm{X}$ & $\mathrm{X}$ & - & - & - & - \\
\hline
\end{tabular}


En el estrato medio las familias Acanthaceae y Cyperaceae, representadas por Bravaisia berlandieriana y Cladium jamaicense respectivamente, fueron las más abundantes en ambos grupos de petenes. En el estrato inferior de los sitios conservados, destacaron en orden de importancia por abundancia las familias Euphorbiaceae, Arecaceae y Ebenaceae con las especies Gymnanthes lucida, Sabal japa y Diospyros verae-crucis, y en los perturbados las familias Acanthaceae, Anacardiaceae y Euphorbiaceae con Bravaisia berlandieriana, Metopium brownei y Gymnanthes lucida.

Los petenes conservados tuvieron menor densidad (ind/ha) y cobertura $\left(\mathrm{m}^{2} /\right.$ ha), en todos los estratos, medio e inferior, respectivamente y mayor área basal $\left(\mathrm{m}^{2} /\right.$ ha) en el estrato superior, que los perturbados (Cuadro 2). Estos últimos concentraron la mayor abundancia de individuos en diámetros $<10 \mathrm{~cm}$ con $80 \%$ del total (Fig. 2a). En orden de importancia aportaron (más de 30\%) a estas clases diamétricas Metopium brownei, Bravaisia berlandieriana, Manilkara zapota y Lysiloma latisiliquum. En los conservados, las especies más abundantes con un diámetro $<10 \mathrm{~cm}$, fueron Metopium brownei, Gymnanthes lucida y Manilkara zapota con 35\% del total de los individuos.

Respecto a la distribución de alturas, los petenes perturbados concentraron 90.5\% de los individuos contabilizados en las dos primeras clases (3-5 m y 5.1-7 m) y una muy baja abundancia en alturas $\geq 10 \mathrm{~m}$. En contraste, los petenes conservados tuvieron mayor uniformidad entre las diferentes clases, contabilizando $52 \%$ entre

Cuadro 2. Comparación de medias y desviación estándar $( \pm)$ para las variables estructurales por grado de perturbación y estratos de vegetación de los petenes estudiados. M-W: Mann-Whitney.

\begin{tabular}{llll}
\hline & Perturbados & Conservados & Análisis \\
\hline $\begin{array}{l}\text { Área muestreo (ha) } \\
\text { Superior }\end{array}$ & 0.46 & 0.54 & Prueba $t^{*} \mathrm{M}-\mathrm{W}$ \\
$\quad$ & & & \\
$\quad \begin{array}{l}\text { Densidad (ind } / \mathrm{ha}) \\
\text { Área basal (m } / \mathrm{ha})\end{array}$ & $438.0 \pm 312.2$ & $223.2 \pm 140.3$ & $p<0.01$ \\
Medio & & $42.5 \pm 31.6$ & $p<0.05$ \\
$\quad$ Densidad (ind $/ \mathrm{ha})$ & $3638.0 \pm 2043.0$ & $1613.0 \pm 1899.0$ & $p<0.01^{*}$ \\
$\quad$ Cobertura (m $/ \mathrm{ha})$ & $912.0 \pm 555.0$ & $503.0 \pm 525.0$ & $p<0.05^{*}$ \\
Inferior & & & \\
$\quad$ Densidad (ind $/ \mathrm{ha})$ & $1346.0 \pm 1397.0$ & $619.0 \pm 561.0$ & $p<0.05$ \\
$\quad$ Cobertura (m $/ \mathrm{ha})$ & $229.8 \pm 269.9$ & $49.4 \pm 65.4$ & $p<0.05$ \\
\hline
\end{tabular}



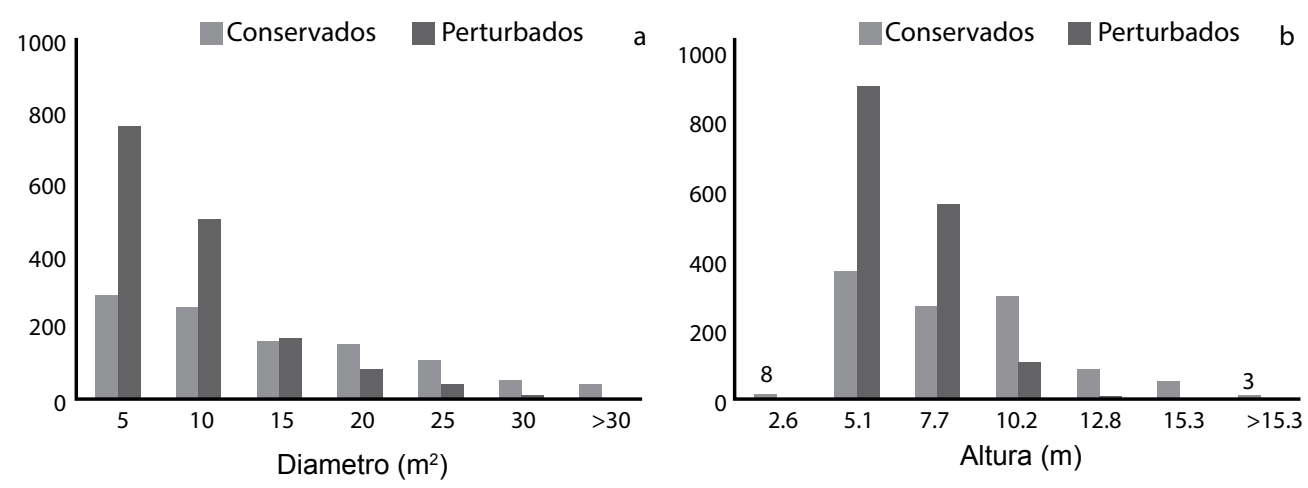

Fig. 2. Distribución de las clases diamétricas (a) y de altura (b) del estrato superior en los petenes conservados y perturbados.

las clases intermedia y alta (Fig. 2b). La distribución en los petenes perturbados refleja el reclutamiento de individuos pequeños debido a la extracción de los grandes.

\section{Suelos}

No se encontraron diferencias significativas en las propiedades del suelo entre los perfiles de los petenes con diferente grado de perturbación. Los suelos en todos los sitios estudiados corresponden a Leptosoles réndzicos, los cuales se caracterizan por tener altos contenidos de materia orgánica en el horizonte superficial y ser limitado en profundidad por material calcáreo. En general, todos los perfiles de suelo, centro y bordo, tuvieron horizontes orgánicos formados a partir de la acumulación de restos vegetales en diferentes grados de descomposición $\left(\mathrm{O}_{1}, \mathrm{O}_{2}\right)$, de alta porosidad, alta abundancia de raíces, de $\mathrm{pH}$ básico (hasta 7.8), alta capacidad de intercambio catiónico, alto contenido de fósforo y nitrógeno total. Subyacente a los horizontes orgánicos se identificó un horizonte $\mathrm{A}$, el cual se distinguió por contener una mezcla de materia orgánica humificada con suelo mineral. Por debajo del horizonte A se identificó un horizonte AC de transición (Cuadro 3), que a su vez yace sobre material calcáreo.

\section{DISCUSIÓN}

La riqueza y diversidad de especies en petenes conservados y perturbados es concordante con tendencias reportadas en estudios realizados por otros autores ( $\mathrm{Du}-$ rán, 1987), los valores más altos se encontraron en los conservados. Esto es similar a 
Cuadro 3. Media y desviación estándar $( \pm)$ de los parámetros de suelo, por horizontes $(\mathrm{H})$ identificados por grado de perturbación; conservados $(\mathrm{C})$ y perturbados $(\mathrm{P})$ de los petenes estudiados. $\mathrm{P}=$ fósforo; M.O. = materia orgánica; D.A. = densidad aparente; C.E. = conductividad eléctrica, $\mathrm{Nt}=$ nitrógeno total, C.I.C. = capacidad de intercambio catiónico.

\begin{tabular}{cccccc}
\hline $\begin{array}{c}\mathrm{H} \\
\text { Prof prom cm }\end{array}$ & & $\begin{array}{c}\mathrm{P} \\
\mathrm{mg} / \mathrm{kg}\end{array}$ & $\begin{array}{c}\mathrm{M} . \mathrm{O} . \\
\%\end{array}$ & $\begin{array}{c}\mathrm{pH} \\
\mathrm{H}_{2} \mathrm{O}(1: 5)\end{array}$ & $\begin{array}{c}\mathrm{D} . \mathrm{A} . \\
\mathrm{g} / \mathrm{ml}\end{array}$ \\
\hline $\mathrm{O}_{1}^{*}$ & $\mathrm{C}$ & $14.99 \pm 6.81$ & $31.97 \pm 5.06$ & $7.56 \pm 0.38$ & $0.36 \pm 0.20$ \\
$0-5.6$ & $\mathrm{P}$ & $23.37 \pm 8.14$ & $30.57 \pm 4.66$ & $7.41 \pm 0.28$ & $0.36 \pm 0.13$ \\
$\mathrm{O}_{2}^{* *}$ & $\mathrm{C}$ & $5.78 \pm 4.49$ & $18.43 \pm 5.70$ & $7.79 \pm 0.11$ & $0.54 \pm 0.12$ \\
$5.6-12.8$ & $\mathrm{P}$ & $12.35 \pm 9.19$ & $20.77 \pm 7.82$ & $7.64 \pm 0.24$ & $0.56 \pm 0.14$ \\
$\mathrm{~A}^{* * *}$ & $\mathrm{C}$ & $2.93 \pm 1.37$ & $6.26 \pm 2.45$ & $8.02 \pm 0.14$ & $0.78 \pm 0.09$ \\
$12.8-24.8$ & $\mathrm{P}$ & $5.06 \pm 7.91$ & $7.79 \pm 7.21$ & $7.73 \pm 0.36$ & $0.60 \pm 0.18$ \\
$\mathrm{AC} * * *$ & $\mathrm{C}$ & $1.97 \pm 0.58$ & $4.55 \pm 3.69$ & $8.08 \pm 0.07$ & $0.88 \pm 0.11$ \\
$24.8-31.3$ & $\mathrm{P}$ & $13.50 \pm 27.34$ & $3.24 \pm 2.47$ & $7.78 \pm 0.45$ & $0.90 \pm 0.11$ \\
\hline $\mathrm{H}$ & & $\mathrm{C} . \mathrm{E}$. & $\mathrm{CaCO}$ & $\mathrm{Nt}$ & $\mathrm{C} . \mathrm{I} . \mathrm{C}$. \\
Prof prom cm & & $\mathrm{dS} / \mathrm{m}$ & $\%$ & $\%$ & $\mathrm{cmol}(+) / \mathrm{kg}$ \\
\hline $\mathrm{O}_{1}^{*}$ & $\mathrm{C}$ & $3.86 \pm 2.38$ & $11.28 \pm 4.68$ & $1.84 \pm 0.29$ & $133.33 \pm 54.48$ \\
$0-5.6$ & $\mathrm{P}$ & $5.36 \pm 2.15$ & $12.60 \pm 1.80$ & $1.76 \pm 0.26$ & $111.17 \pm 26.95$ \\
$\mathrm{O}_{2}^{* *}$ & $\mathrm{C}$ & $7.67 \pm 2.25$ & $16.60 \pm 5.44$ & $0.99 \pm 0.28$ & $46.93 \pm 20.35$ \\
$5.6-12.8$ & $\mathrm{P}$ & $8.01 \pm 1.89$ & $18.04 \pm 3.69$ & $1.19 \pm 0.45$ & $50.19 \pm 24.00$ \\
$\mathrm{~A}^{* * *}$ & $\mathrm{C}$ & $7.27 \pm 1.17$ & $21.26 \pm 0.08$ & $0.36 \pm 0.14$ & $15.49 \pm 4.26$ \\
$12.8-24.8$ & $\mathrm{P}$ & $7.45 \pm 2.11$ & $18.64 \pm 4.16$ & $0.45 \pm 0.42$ & $25.75 \pm 12.83$ \\
$\mathrm{AC}^{* * * *}$ & $\mathrm{C}$ & $4.85 \pm 1.08$ & $17.46 \pm 4.45$ & $0.26 \pm 0.21$ & $8.43 \pm 4.10$ \\
$24.8-31.3$ & $\mathrm{P}$ & $5.04 \pm 2.89$ & $13.39 \pm 7.12$ & $0.19 \pm 0.14$ & $8.86 \pm 8.11$ \\
\hline & & & & &
\end{tabular}

lo observado en el estado de Quintana Roo, México, con respecto a aquellos que han sido impactados por incendios (Sánchez et al., 2007), extracción en selvas húmedas de Australia (Congdon y Herbohn, 1993), y en secuencias de sucesiones forestales de diferentes edades en Brasil (Reiners et al., 1994).

En bosques afectados por extracción en el corto (2.5 años) y largo plazo (50 años) estudiados por Seng et al. (2004), no se observaron cambios en la composición de las especies. En contraste, Álvarez-Yépiz et al. (2008) y Peña-Claros (2003) señalan que la composición y abundancia de especies varía conforme lo hace la edad de bosques manejados, incrementando en número con el paso del tiempo.

En los petenes perturbados, la composición y abundancia relativa de ciertas especies del estrato superior podrían ser tomadas como indicadoras de intervención 
humana. La continua perturbación ha contribuido a una alta abundancia de, por ejemplo leguminosas, similar a lo registrado en otros estudios sobre estadios sucesionales debido a incendios, tumba-roza-quema, y extracción selectiva (Sánchez et al., 2007).

En el presente estudio, las especies de las familias Fabaceae, Mimosaceae y Acanthaceae destacaron en abundancia en relación con las encontradas en petenes conservados. Igualmente aquellas de rápido crecimiento (Metopium brownei, Bravaisia berlandieriana y Lysiloma latisiliquum) que caracterizan los bosques degradados, estudiados por Congdon y Herbohn (1993) y Sánchez et al. (2007), los cuales han indicado que algunas de ellas abundan en las etapas iniciales de la perturbación y permanecen hasta estados avanzados de sucesión. Sin embargo, al término del disturbio es de esperarse el restablecimiento de la composición, dominancia y diversidad de especies a las condiciones originales (Reiners et al., 1994).

Para evaluar las diferencias en la composición de las especies observadas entre los petenes estudiados, debe considerarse el tipo, magnitud, frecuencia e intensidad de las perturbaciones, pues éstas influyen en la disponibilidad de las plantas que estarán recolonizando nuevas áreas, como lo indica Turner et al. (1998).

La composición del estrato medio e inferior mostró que la perturbación ha ocasionado una disminución en las poblaciones de algunas familias como Aracaceae, representada por Sabal japa y Sabal mexicana, plantas que son demandadas y aprovechadas por habitantes de comunidades en la región. Mientras que familias como Acanthaceae representada por Bravaisia berlandieriana, se caracterizaron por ser abundantes en los tres estratos estudiados, lo cual coincide con las observaciones realizadas por Zamora (2003).

Los resultados obtenidos en relación a la densidad y área basal en el presente estudio en general son similares a lo encontrado por Durán (1987) sobre estructura de petenes con dominancia de selva. La disminución de individuos con D.A.P. mayores de $10 \mathrm{~cm}$, que se observó en los sitios perturbados, puede relacionarse con la continua extracción de especies arbóreas de diámetros y alturas de interés para su aprovechamiento. Diversos autores indican que el aprovechamiento selectivo reduce el número de árboles de gran talla (Congdon y Herbohn, 1993; Reiners et al., 1994; Hall et al., 2003). Por ejemplo, Peña-Claros (2003) registró dosel con mayores alturas, conforme se incrementó la edad en bosques manejados de 2 a 40 años en la Amazonia Boliviana, mientras que Álvarez-Yépiz et al. (2008) observaron en bosques secundarios, mayores de 20 años, una área basal $40 \%$ superior a la de un bosque maduro.

El tipo de perturbación también produce efectos diferenciales. Los impactos a la vegetación por fenómenos naturales registrados por Sánchez e Islebe (1999) 
en un sitio de selva mediana subperennifolia indican que, después del paso de un huracán, existe una mayor mortalidad de árboles con diámetros pequeños (3.3-10 $\mathrm{cm}$ ) y una mayor sobrevivencia de árboles en clases diamétricas mayores a $10 \mathrm{~cm}$. Esto contrasta con los resultados obtenidos en el presente estudio y confirma que la perturbación de los petenes estudiados es antrópica.

El incremento observado en el número de individuos en clases diamétricas y altura reducida en los petenes perturbados evidenció que la extracción de especies influye en una alta regeneración, lo que sugiere estado de sucesión temprano. Con el tiempo destacan en abundancia los arbustos y hacia un estado de sucesión más avanzado hay una clara dominancia de individuos arbóreos jóvenes (Gómez-Pompa y Vázquez-Yanes, 1976).

Con base en los resultados de la diversidad de especies, se observó una tendencia entre las dos clases de petenes a diferenciarse estadísticamente. Por lo tanto, se sugiere que futuros trabajos tomen en consideración variables más específicas relativas a la perturbación en el área de estudio que permitan esclarecer esta tendencia. En una investigación realizada por Hall et al. (2003) en un bosque del África Central, no encontraron efecto de la extracción selectiva en la diversidad de especies; esto se atribuyó principalmente al método de muestreo y a la variabilidad natural del bosque.

Los suelos en todos los petenes fueron clasificados como Leptosoles mólicos (Anónimo, 2007). En comparación con Leptosoles de otras regiones de la península de Yucatán, los del sitio de estudio tuvieron mayor contenido de materia orgánica, nitrógeno total, fósforo y capacidad de intercambio catiónico. Con valores considerados de fertilidad óptima (Landon, 1991) para la recolonización de especies vegetales; sin embargo, susceptibles a la degradación fisicoquímica, por oxidación en caso de incendios.

Los altos contenidos de materia orgánica, nitrógeno total, fósforo y capacidad de intercambio catiónico encontrados en los petenes perturbados indican que las actividades humanas, las cuales son principalmente extractivas, a la fecha, no han degradado las condiciones del suelo. El fuego sería la principal amenaza a los suelos; sin embargo, si la quema no es intensa, puede incrementar la cantidad de materia orgánica, fósforo, potasio y $\mathrm{pH}$.

\section{CONCLUSIONES}

Las diferencias encontradas entre petenes en densidad, área basal y cobertura de los diferentes estratos, así como la mayor abundancia de individuos de especies 
arbóreas en clases diamétricas y de altura pequeña en petenes intervenidos pueden ser atribuidas a perturbación antrópica. El impacto por actividades humanas observado en la estructura de la vegetación, por ser de carácter extractivo, no ha afectado las condiciones de fertilidad de los suelos.

\section{AGRADECIMIENTOS}

El primer autor agradece a El Colegio de la Frontera Sur (ECOSUR), al Consejo Nacional de la Ciencia y Tecnología (CONACYT) por la beca asignada (228372) para la realización de los estudios de maestría, así como al Posgrado de ECOSUR por el apoyo para realizar el trabajo de tesis. A Ricardo Góngora Chín y Rodolfo Trejo Noriega, del Herbario del Centro de Desarrollo Sustentable y Aprovechamiento de la Vida Silvestre (CEDESU) de la Universidad Autónoma de Campeche, por el apoyo y dedicación en la identificación de los ejemplares botánicos. A Efraín Aguirre Cortés, por revisión de estilo y formato del manuscrito. A los ayudantes de campo por su apoyo. A César Uriel Romero Herrera, Director de la Reserva de la Biósfera de Los Petenes (2009-2010) por las facilidades y apoyo para realizar las actividades de campo.

\section{LITERATURA CITADA}

Álvarez-Yépiz, J. C., A. Martínez-Yrízar, A. Búrquez y C. Lindquist. 2008. Variation in vegetation structure and soil properties related to land use history of old-growth and secondary tropical dry forests in northwestern Mexico. Forest Ecol. Manag. 256: $355-366$.

Anónimo. 2002. Procedures for soil analysis. 6a. ed. International Soil Reference and Information Centre and Food and Agriculture Organization of the United Nations Technical Paper 9, Wageningen. 120 pp.

Anónimo. 2007. Base referencial mundial del recurso suelo, un marco conceptual para clasificación, correlación y comunicación internacional. Primera actualización 2007. Informes sobre Recursos Mundiales de Suelos No.103. International Union of Soil Sciences, World Soil Information, Food and Agriculture Organization of the United Nations, Roma, Italia. $117 \mathrm{pp}$.

Anónimo. 2009. Guía para la descripción de suelos. 4a ed. Organización de las Naciones Unidad para la Agricultura y la Alimentación (FAO). Traducción de Ronald Vargas Rojas (Proyecto FAO-SWALIM, Nairobi, Kenya-Universidad Mayor de San Simón, Bolivia). Roma, Italia. 99 pp. 
Bray, D. B., E. A. Ellis, N. Armijo-Canto y Ch. T. Beck. 2004. The institutional drivers of sustainable landscapes: a case study of the 'Mayan Zone'in Quintana Roo, Mexico. Land Use Policy 21(4): 333-346.

Congdon, R. y J. Herbohn. 1993. Ecosystem dynamics of disturbed and undisturbed sites in north Queensland wet tropical rain forest. I. Floristic composition, climatic and soil chemistry. J. Trop. Ecol. 9: 349-363.

Chapin, S. F., S. Zavaleta, V. Eviners, R. Naylor, P. Vitousek, H. Reynolds, D. Hooper, S. Lavorel, O. Sala, S. Hobbie, M. Mack y S. Díaz. 2000. Consequences of changing biodiversity. Nature 405: 234-242.

Durán, G. R. 1987. Descripción y análisis de la estructura y composición de la vegetación de los petenes del noroeste de Campeche, México. Biótica 12(3): 181-198.

Flores, J. S. y I. Espejel. 1994. Los tipos de vegetación de la Península de Yucatán. Etnoflora Yucatanense 3: 1-135.

Gentry, A. H. 1988. Changes in plant community diversity and floristic composition on environmental and geographical gradients. Ann. Missouri Bot. Gard. 75: 1-34.

Gómez-Pompa, A. y C. Vázquez-Yánes. 1976. Estudios sobre sucesión secundaria en los trópicos cálido húmedos: El ciclo de vida de las especies secundarias. In: GómezPompa, A., C. Vázquez-Yanes, S. del Amo Rodríguez y A. Butanda Cervera (eds.). Regeneración de selvas. Ed. Continental, México, D.F., México. pp. 559-592.

Hall, S. J., J. D Harris, V. Medjibe y P. M. S. Ashton. 2003. The effects of selective logging on forest structure and tree species composition in a Central African Forest: implications for managements of conservation areas. Forest Ecol. Manag. 183: 249-264.

Landon, J. R. 1991. Booker tropical soil manual: a handbook for soil survey and agricultural land evaluation in the tropics and subtropics. Longman Scientific \& Technical Group. Harlow, UK. 474 pp.

Magurran, A. E. 2006. Measuring biological diversity. Blackwell Publishing. Ames, USA. $256 \mathrm{pp}$.

Mas, J. F. y J. Correa Sandoval. 1999. Análisis de la fragmentación del paisaje en el área protegida "Los Petenes”, Campeche, México. Investigaciones Geográficas. Boletín del Instituto de Geografía, UNAM. 43: 42-59.

Peña-Claros, M. 2003. Changes in forest structure and species composition during secondary forest succession in the Bolivian Amazon. Biotropica 35(4): 450-461.

Reiners, A. W., F. A. Bouwman, J. W. F. Parsons y M. Keller. 1994. Tropical rain forest conversion to pasture: changes in vegetation and soil properties. Ecol. Appl. 4(2): 363-377.

Rico-Gray, V. 1982. Estudio de la vegetación de la zona costera inundable del noroeste del estado de Campeche, México: Los Petenes. Biótica 7(2): 171-203.

Sánchez, O. y G. Islebe. 1999. Hurricane Gilbert and structural changes in a tropical forest in South-Eastern Mexico. Global Ecol. Biog. 8: 29-38.

Sánchez, O., G. A. Islebe y M. Valdez. 2007. Flora arbórea y caracterización de gremios ecológicos en distintos estados sucesionales de la selva mediana de Quintana Roo. Foresta Veracruzana 9(2): 17-26.

Seng, H. W., W. Ratnam, S. M. Noor y M. M. Clyde. 2004. The effects of the timing and method of logging on forest structure in Peninsula Malaysia. Forest Ecol. Manag. 203: 209-228. 
Turner, M. G., W. L. Baker, C. H. J. Peterson y R. K. Peet. 1998. Factors influencing succession: lessons from large, infrequent natural disturbances. Ecosystems 1: 511523.

Turner, B. L., V. S. Cortina, D. Foster, J. Geoghegan, E. Keys, P. Kepleis, D. Lawrence, P. Mendoza, S. Manson, Y. Ogneva-Himmelberger, A. Plotkin, S. D. Pérez, R. Chowdhury, B. Savitsky, L. Schneider, B. Schmook y C. Vance. 2001. Deforestation in the southern Yucatán peninsular region: an integrative approach. Forest Ecol. Manag. 154: 353-370.

Zamora, C. P. 2003. Contribución al estudio florístico y descripción de la vegetación del municipio de Tenabo, Campeche, México. Polibotánica 15: 1-40.

Recibido en agosto de 2012.

Aceptado en septiembre de 2014. 